\title{
Identification of Novel Drug-Like Compounds from Momordica cymbalaria as PPAR- $\gamma$ Agonists: A Molecular Docking Study
}

\author{
E. Abbirami ${ }^{1}$, M. Selvakumar ${ }^{2}$, L. Dinesh Kumar ${ }^{3}$, R. Guna ${ }^{4}$ and T. Sivasudha ${ }^{5^{*}}$ \\ 1,2,3,4\&5 Associate Professor, Department of Environmental Biotechnology \\ Bharathidasan University, Tiruchirappalli, Tamil Nadu, India \\ *Corresponding author \\ E-Mail: sudha@bdu.ac.in, sudacoli@yahoo.com
}

\begin{abstract}
Peroxisome Proliferator-Activated Receptor - $\gamma$ (PPAR $\gamma$ ) is a ligand-activated transcription factor, has become a main target for the treatment of diabetes. It is a member of the nuclear receptor superfamily that regulate the gene expression of proteins involved in glucose, lipid metabolism, adipocyte proliferation and differentiation and insulin sensitivity. Thiazolidinediones (TZDs) are one important class of synthetic agonists of PPAR- $\gamma$. TZDs are antidiabetic agents that target adipose tissue and improve insulin sensitivity, and they are currently being used in the treatment of type 2 diabetes. This work was designed to find out the bioactive compounds from Momordica cymbalaria that have the ability to stimulate the PPAR- $\gamma$ using molecular docking procedure. Six metabolites namely 2-Methoxy-4-vinylphenol, Guaiacol, Carbinoxamine maleate, Azulene, 4N Ethylcytosine and Methyl cinnamate were docked with PPAR- $\gamma$ using AutoDock and the results were determined using binding affinity. Among the six compounds three compounds (Carbinoxamine maleate, 2-Methoxy-4-vinylphenol and 4N Ethylcytosine) showed significant binding affinity towards the PPAR- $\gamma$. Based on the findings of this study these phytochemicals can serve as source of anti-diabetic drugs via agonizing PPAR- $\gamma$.
\end{abstract}

Keywords: Momordica cymbalaria, PPAR- $\gamma$, Anti-diabetic activity, Molecular docking

\section{INTRODUCTION}

Diabetes mellitus is a group of metabolic disorders with a wide variety of etiologies. Diabetes is characterized by hyperglycemia due to impaired insulin secretion, action or both. Mortality and morbidity connected with diabetes are largely attributed to hyperglycemia induced secondary complications such as cardiovascular diseases, nephropathy, neuropathy and retinopathy [1]. Diabetes mellitus is treated mainly by oral anti-diabetic drugs (sulfonylureas, biguanides, alpha-glucosidase inhibitors, dipeptidyl peptidase - 4 inhibitors and thiazolidinediones) which lead to undesirable side effects such as weight gain, renal fluid retention and hypoglycemia. Despite major advancements over the last decade in treating diabetes mellitus, people are unable to manage diabetes efficiently [2].

Many studies have reported peroxisome proliferator activated receptor, as an important regulator of glucose and lipid metabolism. Particularly, PPAR- $\gamma$, the molecular target for thiazolidinediones peroxisome proliferator activated receptor gamma plays a crucial role in the process of insulin sensitization and glucose metabolism. At present, PPAR- $\gamma$ is widely used as a main target in the treatment of diabetes mellitus. The anti-diabetic drugs exert their action by binding to and activating the receptor for PPAR- $\gamma$ which in turn activates the GLUT4 receptor activity [3]. At the same time, natural products and plant extracts have played a major role in the prevention and treatment of diseases and are important sources for drug discovery. Moreover, several medicinal plants are employed for the treatment of diabetes mellitus in India, as in the other parts of the world [4]. Notably, Cucurbitaceae family is considered to be one of the valuable medicinal families of plants with potent hypoglycemic properties. Momordica cymbalaria, a perennial herb from this family is traditionally used to treat diabetes mellitus and has been reported for cardioprotective, hypoglycemic, hepatoprotective, anti-ulcer, anti-microbial and anti-diarrhoeal activity [5]. Recently, this plant has been reported for the presence of bioactive metabolites through GC-MS analysis [6]. Therefore, this study is focused on the identification of potent compound from Momordica cymbalaria which can be used as an agonist for PPAR- $\gamma$.

\section{MATERIALS AND METHODS}

\section{A. Protein Preparation}

The 3D structure of the nuclear transcriptional factor, PPAR gamma (PDB ID: 2HFP) was downloaded from the protein data bank (http://www.rcsb.org/pdb/home/home.do) to carry out docking simulations. All non-protein molecules were eliminated from 2HFP using Discovery Studio Version 2017. (Fig. 1A)

\section{B. Ligand Preparation}

The compounds were obtained from our previous work that reported the presence of bioactive compounds in Momoridca cymbalaria [6] and the 3D structure of ligand for this docking study was downloaded from PubChem in the sdf file format. The compound chemical structure files were converted in to PDBQT format using AutoDock 4.0.

\section{Drug Likeness Property}

Drug likeness of all the phytocompounds were predicted using DruLito property prediction tool [7]. This tool analyses the molecular properties of drug candidates based on Lipinski's rule of five and examines the violation of 
distinct properties. Below are the points that represents Lipinski's rule of five

1. Molecular Weight less than 500 Dalton

2. $\log \mathrm{P}$ value less than 5

3. Less than 5 hydrogen bond donors

4. Less than 10 hydrogen bond acceptors

5. Molar refractivity should be between $40-130$

\section{Active Binding Site Prediction}

The active binding sites of PPAR $\gamma$ were predicted by RaptorX-Binding (http://raptorx.uchicago.edu/BindingSite/) which helpful to make grid box preparation on docking studies $[8,9,10]$.

\section{E. Molecular Docking}

Protein-ligand docking approach was used to analyze structural complexes of PPAR- $\gamma$ with bioactive compounds of Momoridcacymbalaria, in order to understand the structural basis of this protein target specificity. Molecular docking was carried out by AutoDock 4.0 version based on scoring functions. Initially, the pdb file of protein structure (2HFP) was converted into PDBQT format and the grid box was prepared for docking analysis. The binding affinity of the ligand to the protein was computed using the AutoDock 4.0 by Lamarckian Genetic searching Algorithm. Evaluation of docking results were sorted via predicted binding energy (kcal/mol). A cluster analysis based on root mean square deviation values (RMSD values) and the lowest energy conformation of the most populated cluster was considered as the most trustable solution.

\section{F. Visualization}

The AutoDock results were analyzed using Discovery Studio Version 2017. The different binding mode of ligands file obtained from the end of the docking studies and that files were analyzed on Discovery Studio to check the best binding mode with the binding site cavity. The proteinligand interactions of $\mathrm{H}$-bond, $\mathrm{CH}$-bond, Vander Waals attraction, Pi-Alkyl, Alkyl and Pi-Pi Alkyl interactions were viewed and various atomic distance, angle were measured the same software.

\section{RESULTS AND DISCUSSION}

Totally, 23 compounds from GC-MS profiling of Momordica cymbalaria were selected for the current docking study. Drug likeness of those compounds was investigated before docking with PPAR $\gamma$. Peroxisome proliferator-activated receptors, plays a main role in controlling, expression of gene involved in adipogenesis, lipid metabolism, inflammation, and the maintenance of metabolic homeostasis. Moreover, coordinates the pathways involved in lipid and glucose metabolism. Based on the previous reports, it is clear that, $\operatorname{PPAR} \gamma$ is the bestcharacterized member of the PPAR $\gamma$ family; it is predominantly expressed in adipose tissue and plays a significant role in lipid metabolism, adipogenesis, glucose homeostasis, and insulin sensitization. Various molecules have been suggested as biological ligands of PPAR $\gamma$ : polyunsaturated fatty acids, prostanoids, eicosanoids, components of oxidized low-density lipoproteins and oxidized alkyl phospholipids [11].

\section{A. Drug Likeness Property}

Initially, 23 compounds from Momordica cymbalaria were analyzed using DruLito. This tool selects the drug like compounds which follows the Lipinski's Rule of five (molecule with a molecular weight less than $500 \mathrm{Da}$, no more than 5 hydrogen bond donors, less than 10 hydrogen bond acceptors, an Octanol-water partition co-efficient log $\mathrm{P}$ less than 50). 2-Methoxy-4-vinylphenol, Guaiacol, Sarcosine, Carbinoxamine maleate, Propanamide, Ethyl Butyrate, Deanol, Azulene, Butyl formate, 2-Azetidinone, $4 \mathrm{~N}$ Ethylcytosine and Methyl cinnamate are the 12 compounds chosen for further docking analysis based on the results provided by DruLito (Table I).

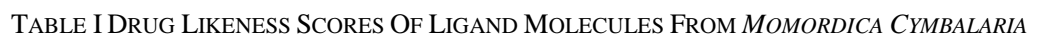

\begin{tabular}{|c|l|c|c|c|c|c|c|}
\hline S. No. & \multicolumn{1}{|c|}{ Compound } & Log P & No. of RB & $\begin{array}{c}\text { No. of } \\
\text { HBA }\end{array}$ & $\begin{array}{c}\text { No. of } \\
\text { HBD }\end{array}$ & TPSA & AMR \\
\hline 1 & 2-Methoxy-4-vinylphenol & 1.83 & 2 & 2 & 1 & 29.46 & 48.13 \\
\hline 2 & Guaiacol & 0.975 & 1 & 2 & 1 & 29.46 & 39.2 \\
\hline 3 & Sarcosine & -0.794 & 2 & 3 & 2 & 49.33 & 20.78 \\
\hline 4 & Carbinoxamine maleate & 0.745 & 6 & 3 & 0 & 24.83 & 90.63 \\
\hline 5 & Propanamide & -0.555 & 1 & 2 & 1 & 43.09 & 17.89 \\
\hline 6 & Ethyl Butyrate & 1.486 & 4 & 2 & 0 & 26.3 & 29.64 \\
\hline 7 & Deanol & -0.646 & 2 & 2 & 1 & 23.47 & 26.28 \\
\hline 8 & Azulene & 3.224 & 0 & 0 & 0 & 0 & 49.15 \\
\hline 9 & Butyl formate & 1.355 & 4 & 2 & 0 & 26.3 & 24.08 \\
\hline 10 & 2-Azetidinone & -0.67 & 0 & 2 & 1 & 29.1 & 16.7 \\
\hline 11 & 4N Ethylcytosine & 0.102 & 2 & 4 & 2 & 53.49 & 36.37 \\
\hline 12 & Methyl cinnamate & 2.208 & 3 & 2 & 0 & 26.3 & 51.97 \\
\hline
\end{tabular}


Log P- Logarithm of partition coefficient, RB -Rotatable Bonds, HBA- H-Bond Acceptor, HBD- H-Bond Donor, TPSA- Total Polar Surface Area, AMR - Atom Molar Refractivity.

\section{B. Active Binding Site Prediction}

The possible active binding sites of PPAR- $\gamma$ were predicted from RaptorX-Binding online server. The binding sites of PPAR-Gamma is phe303, ile320, phe321, gly323, cyc324, gln325, arg327, ser328, his362, ile365, tyr366, lys367, leu369, val378, ile380, ser381, met387, leu392, phe402, met403, his488, leu508, tyr512.

\section{Molecular Docking}

Molecular docking is one of the major domains of drug designing which predicts the structure of intermolecular complex formed between the target and ligand molecules. It is widely used to predict the different binding modes of ligand molecules with the drug targets.AutoDock 4.0 was used to calculate the ligand binding to PPAR $\gamma$. The results acquired from AutoDock were analyzed to study the binding energy and the interaction of the docked structure. 12 compounds with druglikeness property retrieved from DruLito were docked with PPAR- $\gamma$ using AutoDock 4.0.

The protein ligand complexes formed using least binding energy are considered to have highest binding affinity.Six compounds (2-Methoxy-4-vinylphenol, Guaiacol, Carbinoxamine maleate, Azulene, 4N Ethylcytosine and Methyl cinnamate) with lesser binding energy when comparing the other drug like compounds from Momordica cymbalaria, were selected for further interaction studies (Table II).

TABle Ii Binding ENERgies Of Ligand Molecules With PPAR- $\gamma$, PERFORMED USING AUTODOCK 4.0

\begin{tabular}{|c|l|c|}
\hline S. No. & \multicolumn{1}{|c|}{ Compounds } & $\begin{array}{c}\text { Binding } \\
\text { Energykcal/mol }\end{array}$ \\
\hline 1 & $\begin{array}{l}\text { 2-Methoxy-4- } \\
\text { vinylphenol }\end{array}$ & -5.6 \\
\hline 2 & Guaiacol & -5.2 \\
\hline 3 & Sarcosine & -0.38 \\
\hline 4 & $\begin{array}{l}\text { Carbinoxamine } \\
\text { maleate }\end{array}$ & -6.7 \\
\hline 5 & Propanamide & -3.7 \\
\hline 6 & Ethyl Butyrate & -4.7 \\
\hline 7 & Deanol & -3.4 \\
\hline 8 & Azulene & -5.5 \\
\hline 9 & Butyl formate & -4.1 \\
\hline 10 & 2-Azetidinone & -3.6 \\
\hline 11 & 4N Ethylcytosine & -5.5 \\
\hline 12 & Methyl cinnamate & -5.5 \\
\hline
\end{tabular}

\section{Interaction studies}

PPAR $-\gamma$ is acting as a target for insulin sensitizing target, which enhances plasma glucose level in diabetic people.Thiazolidinediones (TZDs) are one important class of synthetic agonists of PPAR $\gamma$. TZDs are antidiabetic drugs currently used in the treatment of type 2 diabetes mellitus that target adipose tissue and improve insulin sensitivity. However; the administration of TZDs has been linked with adverse side effects such as fluid retention, weight gain, cardiac hypertrophy, bone fractures, and hepatotoxicity. Hence, there is a need to search for a new peroxisome proliferator-activated receptor gamma agonist with little or no side effect. Several PPAR $\gamma$ ligands were found in natural products that are common food sources, including the tea plant, soybeans, palm oil, ginger, grapes, spices and wine [12]. A similar study by Prabhu et al., 2011 [13], demonstrates the docking and interaction motifs of punigluconin, Niranthin and emblicanin B with PPAR $\gamma$. Among the six phytocompounds docked against PPAR $\gamma$, compounds that possesses binding energy greater than -5.0 were visualized under Discover study 2017.
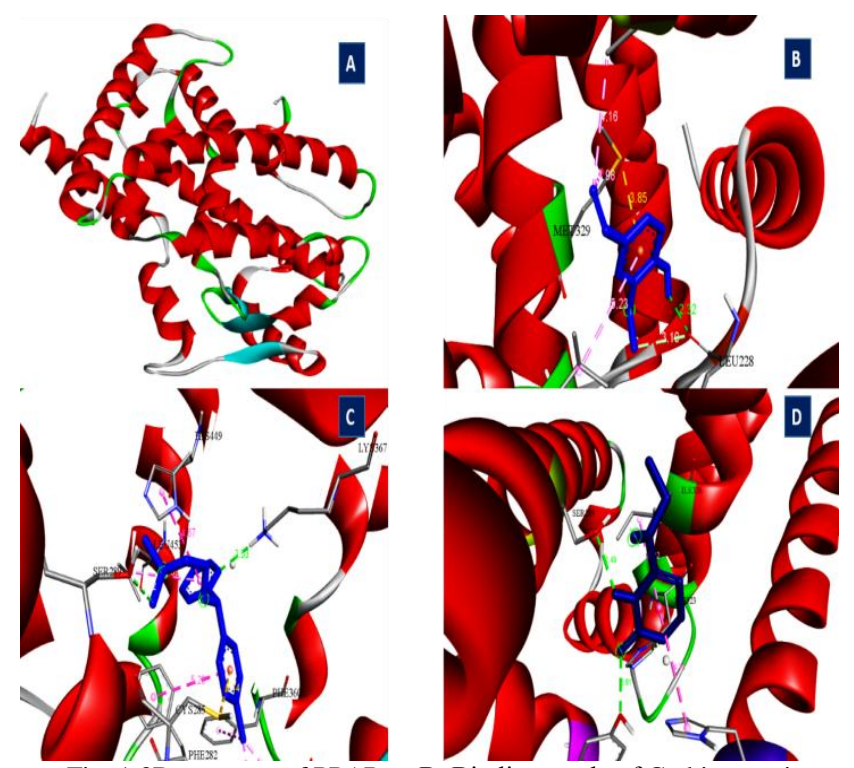

Fig. $13 \mathrm{D}$ structure of PPAR- $\gamma$, B. Binding mode of Carbinoxamine maleate with PPAR- $\gamma$, C. Binding mode of 2-Methoxy-4-vinylphenol, D. Binding mode of $4 \mathrm{~N}$ Ethylcytosine with PPAR- $\gamma$

As a result, carbinoxamine maleate predominantly, showed the highest binding affinity $(-6.7 \mathrm{kcal} / \mathrm{mol})$ with PPAR- $\gamma$ which was evident from the one $\mathrm{H}$-bond interaction (Lys367: $2.52 \AA$ ), one carbon hydrogen interaction (Ser289: $3.52 \AA$ ) and 6 hydrophobic interactions (Cys285: $5.13 \AA$; Phe282: $5.22 \AA$; His449: $4.66 \AA$; Ile281: $4.6 \AA$; Leu356: $5.22 \AA$, Phe360: $4.69 \AA$ A) (Fig. 1B). 2-Methoxy-4vinylphenol and $4 \mathrm{~N}$ Ethylcytosine are the two other compounds that showed better interactions than the other docked compounds. 2-Methoxy-4-vinylphenol- PPAR- $\gamma$ complex displayed one conventional H-bond interaction (Leu228: $2.31 \AA$ ), one carbon hydrogen interaction (Leu228: $3.09 \AA$ ) and 3 hydrophobic interactions (Ala292: $4.15 \AA$; Met: $3.97 \AA$; Leu333: $5.22 \AA$ ) (Fig. 1C). 
The 4N Ethylcytosine - PPAR- $\gamma$ complex showed the 2 hydrogen bond interaction (Ser289: $2.49 \AA$; Tyr473: 2.81 $\AA$ ), one carbon H-bond interaction (His323: $3.49 \AA$ ), and 2 hydrophobic interactions (His449: $4.69 \AA$; Ile326: $5.31 \AA$ ) (Fig. 1D). Particularly, docking results showed that, Carbinoxamine maleate was tightly bound to the active binding site of Lys367 with $2.52 \AA$ distance towards PPAR$\gamma$. From the above results, it was concluded that the phytocompounds present in Momordica cymbalaria namely, Carbinoxamine maleate, 2-Methoxy-4-vinylphenol and 4N Ethylcytosine could influence nuclear transcriptional factor, PPAR- $\gamma$ because of their higher interaction binding affinities with minimized binding energy.

\section{CONCLUSION}

Carbinoxamine maleate, 2-Methoxy-4-vinylphenol and 4N Ethylcytosineare are the three ligand molecules from Momordica cymbalaria that showed significant docking scores and interaction patterns with PPAR- $\gamma$. Therefore, those compounds can be considered as promising candidates for the development of novel anti-diabetic drugs. However, further cellular and in vivo investigations are needed to confirm the in silico findings.

\section{REFERENCES}

[1] E. Ozturk, A.K.K. Arslan, M.B. Yerer, and A. Bishayee, "Resveratol and diabetes: a critical review of clinical studies", Biomed. Pharmacother, Vol. 5, pp. 230-234, 2017.

[2] J.H. He, L.X. Chen, and H. Li, "Progress in the discovery of naturally occurring anti-diabetic drugs and in the identification of their molecular targets", Fitoterapia, Vol. 134, pp. 270 - 289, 2019.
[3] Tenenbaum, E.Z. Fisman, and M. Motro, "Metabolic syndrome and type 2 diabetes mellitus: focus on peroxisome proliferator activated receptors (PPAR)", Cardiovascular diabetology, Vol. 2, No. 1, pp.4, 2003.

[4] R. Jeyadevi, T. Sivasudha, A. Rameshkumar, B. Sangeetha, D. Arul Ananth, and G. Smilin Bell Aseervatham, " Nutritional constituents and medicinal values of Momordica cymbalaria (Athalakkai) - A review", Asian Pac J Trop Biomed., pp. S456-S461, 2012.

[5] H.Y. Hung, K. Qian, S.L. Morris-Natschke, C.S. Hsu and K.H. Lee, "Recent discovery of plant-derived anti-diabetic natural products", Nat. Prod. Rep., Vol. 2, No.5, pp.580-606, 2012.

[6] E. Abbirami, L. Dinesh Kumar, R. Guna, N. Gayathri, and T. Sivasudha, "Alpha amylase, alpha glucosidase inhibition and profiling of volatile compounds of biologically active extracts from Momordica cymbalaria (Hook, Fenzl) skin and seeds", AJEAT, Vol. 8, No. 1, pp. 67-73, 2019.

[7] G.R. Bickerton, G.V. Paolini, J. Besnard, S. Muresan, and A.L. Hopkins, "Quantifying the chemical beauty of drugs", Nat. Chem., Vol. 4, No. 2, p.90, 2012.

[8] J. Ma, S. Wang, F. Zhao, and J. Xu, "Protein threading using contextspecific alignment potential", Bioinformatics., Vol. 29, No. 13, pp. 257- 265, 2013.

[9] M. Kallberg, H. Wang, S. Wang, J. Peng, Z. Wang, H. Lu, and J. Xu, "Template-based protein structure modeling using the RaptorX web server", Nature protocols., Vol.7, No. 8, pp.1511, 2012.

[10] J. Ma, J. Peng, S. Wang and J. Xu, "A Conditional Nueral Fields model for protein threading", Bioinformatics, Vol. 28, No. 12, pp.59 66, 2012.

[11] M.A. Lee, L. Tan, H. Yang, Y.G. Im, and Y.J. Im, 2017, "Structures of PPAR $\gamma$ complexed with lobeglitazone and pioglitazone reveal key determinants for the recognition of antidiabetic drugs", Scientific reports., Vol.7, No.1, pp.16837, 2017.

[12] L. Wang, B. Waltenberge, and E.M. Pferschy-Wenzig, "Natural product agonists of peroxisome proliferator-activated receptor gamma (PPARgamma): a review", BiochemPharmacol., Vol. 92, No.1, pp. 73-89, 2014.

[13] S. Prabhu, S. Vijayakumar, P. Manogar, G.P. Maniam, and N. Govindan, N., "Homology modeling and molecular docking studies on Type II diabetes complications reduced PPAR $\gamma$ receptor with various ligand molecules", Biomed. Pharmacother, Vol. 92, pp. 528$535,2017$. 